\title{
MODE LOCALIZATION BY STRUCTURAL IMPERFECTIONS IN ONE-DIMENSIONAL CONTINUOUS SYSTEMS
}

\author{
A. LUONGO \\ Dipartimento di Ingegneria delle Strutture, Università di L'Aquila, \\ Monteluco di Roio, 67040 L'Aquila, Italy
}

(Received 18 April 1990, and in revised form 3 April 1991)

\begin{abstract}
Longitudinal free oscillations of a beam with small axial rigidity, continuously restrained by imperfect elastic springs, are analyzed. It is shown that small imperfections can localize the first modes of vibration in a restricted region of the beam. Systems with single localized defects are first considered; these provide physical insights and make clear that the phenomenon is governed by a turning point mathematical problem. Analogies with similar problems in quantum mechanics are emphasized. Periodic or nearly periodic imperfections are then analyzed by applying the Floquet theory, and finally non-periodic imperfections are numerically treated. It is shown that when the exponential decay of the solution is strong, the modes localize in the neighbourhood of each defect, so it is possible to limit oneself to a local analysis. The problem of a beam of infinite length with a single imperfection is then solved by applying the asymptotic WKB method. An application relative to a parabolic imperfection is analytically developed.
\end{abstract}

\section{INTRODUCTION}

The localization phenomenon of vibrations in mechanical systems has received considerable attention in the past few years. It has been shown that, under certain conditions, the mechanical behaviour of particular systems, such as periodic structures made up of assemblies of equal substructures, can be considerably influenced by the presence of small imperfections. In particular, the normal modes of oscillation, which in the absence of defects are extended to the whole system (overall modes), can localize in a restricted region and assume the character of local modes. Consequently, the energy injected into the system cannot propagate very far but is confined to a region close to the source. Therefore localization has an effect similar to damping, since it inhibits propagation of motion, albeit through confinement of the energy rather than by dissipation.

The phenomenon was first studied in solid state physics, and in a famous paper Anderson [1] explained many of the transport properties of disordered solids; this aroused considerable interest. The motion of electrons in solids is governed by the Schrödinger wave equation: if the eigenmodes are extended, metallic conduction is allowed, if they are localized conduction is forbidden. This means that solids with a regular crystal lattice exhibiting metallic behaviour may become semiconductors when this order is destroyed by imperfections.

Anderson introduced a simple model of cellular disorder, considering atoms situated at lattice sites with next-neighbour interactions only and site energies as independent stochastic variables uniformly distributed. He showed that the wave functions of this model decay exponentially on the average away from the centre of localization. There is a great amount of literature on the subject; here it is sufficient to mention only the paper by Herbert and 
Jones [2] who obtained improved conditions in Anderson's model by assuming specific correlations amongst the levels, and the extensive review by Erdös and Herndon [3] treating the questions of the density electron states in one or more dimensions and transmission through a sequence of potentials.

Since the whole matter bears a strong analogy to the mechanical problem referred to above, in order to avoid misunderstandings between physicists and engineers, it is advisable to make a few preliminary comments. The strong analogy stems from the fact that the time independent Schrödinger equation and the time harmonic wave equation have precisely the same mathematical form. Thus, many results achieved in solid state physics could be applied directly to structural dynamics. Nevertheless, as stressed in references [4] and [5], there are some basic aspects that render the two problems different and justify a "strictly mechanical" approach. First, (a), in structural engineering, boundary conditions play an important role in defining the behaviour of the system; on the contrary, in atomic models of crystalline solids, large numbers of subsystems are present, and the effects of boundary conditions are negligible. Second, (b), such a large number of units suggests a statistical approach in theoretical physics, whereas a deterministic approach retains its validity in structural mechanics. Third, (c), most atomic models deal with lumped masses separated by comparatively large distances, whereas structures usually have distributed masses. Fourth, (d), large defects are often deliberately introduced in crystalline solids by doping them, whereas in engineering small irregularities are usually due to manufacturing and material tolerances.

As a result of these intrinsic differences of the objects to be studied, physicists and engineers have very different objectives regarding localization. Indeed, one of the most important aims in physics is to prove the existence of spatial decay due to disorder, not to calculate the rate of spatial decay. This stems from the fact that however small the decay may be, it is sufficient to inhibit propagation through the lattice if this is long enough. On the contrary, in structural dynamics it is important to determine the exponential rate of decay (the so-called localization factor [5]) because it is responsible for the strength of localization effects. Due to the relatively small number of subsystems (typically a few hundred), the localization phenomenon is important in structural dynamics only if the subsystems are weakly coupled. In this case a small amount of disorder gives rise to strong localization, in the meaning ascribed to it by Pierre [5], Pierre and Dowell [6], and Pierre and Cha [7]. On the contrary, weak localization (according to Kissel [8, 9]), which occurs when the number of the substructures is large and the coupling is not small, is important in physics but is of little interest in engineering. For the reasons indicated above, the term "localization" will be used in the sense of "strong localization" and the term "Anderson localization" will be avoided, so as not to cause confusion with the original terminology adopted in physics. Certain other differences and analogies between the two problems are emphasized in the paper.

If normal modes are to localize two conditions must be satisfied: (1) the system must be at high modal density, i.e., numerous frequencies must be contained in a narrow band; (2) some disorder must exist in the system. Under these conditions, marked changes in response are associated with small local variations in geometrical, mechanical and inertial properties: the system is sensitive to the initial imperfections.

The imperfection sensitivity concept is well known in static stability problems, since Koiter [10] introduced it in 1945, and after recent advances due to the modern catastrophe theory, but it has not yet been made clear in dynamic problems. A contribution has been made in this regard by Bendiksen [11] who discussed the application of a regular perturbation method to systems at high modal density, giving an explanation of the mechanism producing the localization. A regular perturbation method was presented by Pierre and 
Dowell [6] and successively utilized in references $[7,12,13]$ after taking up an idea contained in a previous paper by Hodges and Woodhouse [14]. The method provides important insights into mode localization and permits derivation of criteria to predict the occurrence of the phenomenon. A statistical approach for calculating the sensitivity of characteristic roots to parameter uncertainties was presented by Pierre [15]; a review of the subject can be found in reference [16].

With but a few exceptions, all the literature on mechanical localization in recent years refers to discrete one-dimensional models, such as open or closed chains of pendulums $[5,6,11,13,17-19]$. Continuous systems, such as chains of beams $[7,20]$, multi-span beams [11-13] or taut strings with attached lumped masses [14], are discretized through finite elements or Galerkin techniques. In most of the papers the problem is dealt with via modal analysis, and in only a few via travelling waves $[17,21]$. The approach is often deterministic and only recently has it become probabilistic $[5,8,9,21]$. Then again, in some papers continuous periodic systems are considered without discretizing $[4,8,9]$. However, the periodicity of the problem reduces the unknowns to a finite number: e.g., if use is made of the transfer matrices.

To the author's knowledge, the only paper in which localization in a continuous model is considered by means of an analytical approach is that by Scott [22], who considered the one-dimensional scattering of waves in a time independent random medium by using stochastic process theory to investigate the statistics of the wavefield. He showed that the effects of localization appear in the statistical properties of wave intensity as exponential decay factors multiplying a fluctuating component. In particular, he emphasized that the use of average variables can be misleading, because of the long tail of the log-normal distribution. Thus, the average value of the intensity is heavily weighted by exceptional configurations, and does not represent typical behaviour of members of the ensemble. This last problem has been more recently reconsidered and discussed in depth by Hodges and Woodhouse $[18,21]$.

The localization phenomenon in continuous one-dimensional systems is considered again here, albeit from a different angle (see also reference [23]). The structural model investigated is composed of an axially vibrating beam, with small extensional stiffness, continuously restrained by longitudinal elastic springs, devoid of equal stiffness because of the presence of imperfections. The model can be considered as limit of a chain of fixed length, made of subsystems each with one degree of freedom, when the number of substructures increases to infinity. Thus the distributed mass of the beam and the elastic constraints constitute the substructures, while the small axial stiffness of the beam produces the coupling between them. Closed form solutions are obtained, relevant to a single defect of stiffness or to periodic and nearly periodic diffused imperfections, as well as numerical solutions relevant to non-periodically diffused imperfections. They show that the localization phenomenon can be considered as a classical mathematical physics turning point problem. A parametric analysis highlights of the peculiar aspects of the phenomenon and its dependence on boundary conditions, as well as the shape and position of the imperfections. By taking into account these results, an application of the asymptotic WKB method of solving the turning point problem is indicated.

There are many differences between the present approach to the problem and Scott's study: (a) in reference [22] the vibration problem is solved in terms of travelling waves, here by means of normal modes; (b) the domain of integration is semi-infinite in reference [22], finite in the present analysis; (c) the problem is solved in a probabilistic context in reference [22] and in a deterministic way in this paper. However, these are only methodological aspects, the most important difference being the following. (d) The continuous elastic constraint considered here is absent in Scott's work. Therefore Scott's model can 
be considered as the continuous counterpart of a spring-mass chain, while the present model is the counterpart of a one-degree-of-freedom chain. As discussed by Pierre [5], the first model can highlight only weak localization, because it does not allow variation of the relative magnitudes of the internal coupling and disorder, whereas the second model permits the study of both weak and strong localization.

The main aims of this paper are as follows: (1) to clarify the understanding of the mechanical phenomenon; (2) to show that the simple continuous model permits the acquisition of analytical results hitherto obtained in literature only numerically for the corresponding discrete model; (3) to emphasize that localization is closely connected with the occurrence of turning points in the solution, so that the phenomenon can be correctly placed in a class of problems already studied from an analytical point of view. To this end the problem is solved in a deterministic context which, although unsuitable for describing the physical reality, allows explanation of the basic phenomena in a very simple way. Indeed, even if the term Anderson localization refers typically to a random spatial distribution of disorder, whereas only one or a few defects are considered in this paper, it is held here that the basic physical mechanism that produces confinement is the same in both cases.

The work described here is the result of research carried out in parallel with the analysis of the amplitude modulation and localization phenomena which occur in some buckling problems in the post-critical range [24, 25] (mode localization in linear buckling problems has also been studied by Pierre [26]). In particular, in reference [25] it was emphasized that the geometric non-linearities present in a class of systems with high modal density have the same effect, with regard to the localization, that the imperfections have in linear dynamics; indeed, they again lead to a turning point problem. The two problems therefore exhibit close analogies that should be further analyzed in depth.

\section{STRUCTURAL MODEL}

A study is made of longitudinal oscillations of a beam of length $l$, small axial stiffness $E A$ and mass per unit length $m$, continuously restrained by longitudinal springs of stiffness $k$ (see Figure 1). The normal modes of oscillations $u(x)$ of circular frequency $\omega$, satisfy the differential equation

$$
E A u^{\prime \prime}-k u+m \omega^{?} u=0 .
$$

Let us consider first the ideal case of a perfect system, characterized by values of $E A, K$ and $m$ constant along the beam. Here the modes of vibration are periodic functions defined on the whole beam and are referred to as extended or collective. When the stiffness of the beam approaches zero, the modes remain periodic, however small, but different from zero, $E A$ is. For $E A=0$ and $m \neq 0$ the system degenerates into an infinite number of independent one-degree-of-freedom systems.

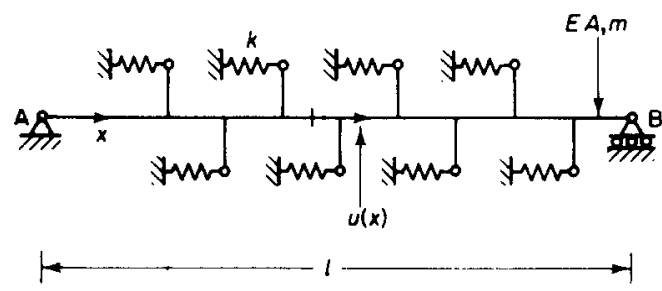

Figure 1. Structural model. 
Let us consider now the real case of an imperfect system, characterized by small structural irregularities, such as weak variations along the $x$-axis of the mechanical and inertial properties. Here the case of springs of variable stiffness $k$ is considered. Due to these imperfections the vibration modes differ from those of the perfect system; in particular, they can lose the character of periodicity and be localized or confined in a small region of the beam. It is wished to analyze the conditions under which this phenomenon occurs.

Equation (1) can be rendered dimensionless as

$$
\beta u^{\prime \prime}+(\lambda-\kappa(x)) u=0,
$$

where

$$
\beta=E A / k_{0} l^{2}, \quad \lambda=m \omega^{2} / k_{0}, \quad \kappa(x)=k(x) / k_{0} .
$$

In equations (2) and (3) $k_{0}$ is the constant nominal value of the stiffness of the springs with $u=u / l$ and $x=x / l$; a prime denotes differentiation with respect to the dimensionless variable. Note that $\beta \ll 1, E A$ being small by hypothesis, and $\kappa=O(1)$, the imperfections being small too. In addition, due to the high modal density, $\lambda=O(1)$ for the first modes, since $\omega$ is very close to $\sqrt{k_{0} / m}$. Moreover, it is convenient to put

$$
\kappa(x)=1+\gamma \chi(x),
$$

where $\chi(x)$ describes the imperfection shape, with $|\chi|_{\max }=1$, and $0<\gamma \ll 1$ measures its amplitude.

It is worth noting that equation (2) is formally identical to the one-dimensional time independent Schrödinger equation [27-29], which in quantum mechanics governs the steady state motion of a particle, if the following identifications are made: $u(x)$ is the wave function that measures the probability density of finding the particle at the abscissa $x$; the eigenvalue $\lambda$ is the non-dimensional total energy of the particle; the function $\kappa(x)$ is the non-dimensional potential energy of the external field, assumed to depend only on position; the small parameter $\beta$ is proportional to the squared Planck's constant. Thus, results already known in quantum mechanics will be obtained again here but with reference to the steady state motion of the axially vibrating beam.

\section{SINGLE LOCALIZED IMPERFECTION}

Equation (2) can be easily integrated in the particular case of a piecewise constant function $\chi(x)$. Here the simplest case of imperfection is considered, sufficient to explain the fundamental aspects of the phenomenon.

\subsection{THEORY}

Let the beam be fixed at the ends, with a unique defect of stiffness localized at midspan, of extension $2 \eta$ and amplitude $\gamma$ (see Figure 2). Due to symmetry it is possible to

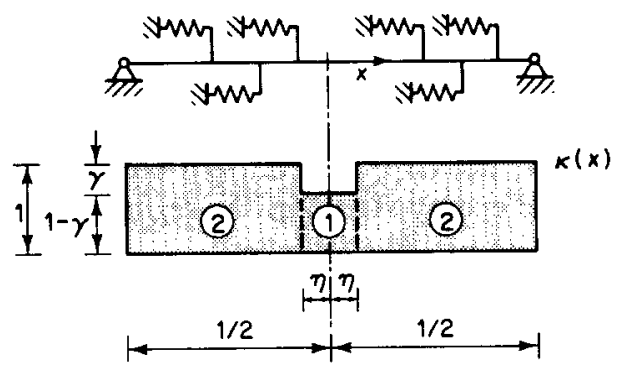

Figure 2. Impulsive defect of stiffness. 
study the problem in the interval $[0,1 / 2]$, in which equation (2) becomes

$$
\left\{\begin{array}{ll}
\beta u_{1}^{\prime \prime}+(\lambda-1+\gamma) u_{1}=0 & \text { for } 0 \leqslant x<\eta \\
\beta u_{2}^{\prime \prime}+(\lambda-1) u_{2}=0 & \text { for } \eta<x \leqslant 1 / 2
\end{array}\right\} .
$$

In order to solve equations (5) it is necessary to distinguish two cases: (a) for $\lambda>1$ the functions $u_{1}(x)$ and $u_{2}(x)$ are periodic with different periods so that modal shapes are extended; (b) for $1-\gamma<\lambda<1, u_{1}(x)$ is periodic while $u_{2}(x)$ is exponential, so the mode is localized near the defect; the character of the solution changes at the point $x=\eta$, which is called the turning or transition point [30]. The case $\lambda<1-\gamma$ has no solution, as is easy to see by applying Rayleigh's theorem.

These considerations suggest the following simple interpretation of the localization phenomenon. Let us consider first the degenerated system, formed of equal springs and an unstiff beam; in this case the Dirac function is an eigenfunction and $\lambda=1$ is an eigenvalue of infinite multiplicity. When the small stiffness of the beam is introduced (perfect system), all frequencies are split and become of order $\lambda=O\left(1+n^{2} \beta\right), n$ being the mode number, while the eigenfunctions become extended and periodic. Let us now introduce a defect of stiffness (imperfect system) : frequencies decrease and in particular the first ones-which are slightly greater than 1 if $\beta$ is small-can become smaller than 1 , thus entailing localization. Therefore, only first modes can localize, and their number increases for decreasing stiffness of the beam and increasing amplitude of the imperfection.

Determination of the solution to equations (5) is straightforward and is not reported here; details can be found in reference [23]. It is useful, however, to make some comments. The real or imaginary parts of the exponential factors of eigenfunctions $u_{1}(x), u_{2}(x)$, from equations (5) respectively are seen to be

$$
\theta_{1}=\sqrt{(\lambda-1+\gamma) / \beta}, \quad \theta_{2}=\sqrt{|\lambda-1| / \beta},
$$

and the following relationships hold good, where the upper sign refers to case (a) and the lower to case (b):

$$
\theta_{1}^{2} \mp \theta_{2}^{2}=\gamma / \beta \text {. }
$$

From equation (7) these conclusions can be drawn:

(a) Non-localized modes $(\lambda>1)$. If the ratio $\gamma / \beta$ is small (small imperfection, large axial stiffness) $\theta_{1}$ and $\theta_{2}$ are nearly equal, and the imperfection produces only a feeble reduction of the wavelength in the region of the defect. The reduction of the amplitude can instead be large, but is restricted to a small region of the beam. On the contrary, if $\gamma / \beta$ is large (large imperfection, small stiffness), $\theta_{1}$ and $\theta_{2}$ are very different.

(b) Localized modes $(\lambda<1)$. From equation (7) it is seen that $\theta_{1}$ and $\theta_{2}$ range between zero and $\sqrt{\gamma / \bar{\beta}}$. The first localized modes are associated with smaller values of $\theta_{1}$ and consequently with greater values of $\theta_{2}$; therefore they are more strongly localized than higher modes. Because of $\theta_{1}<\sqrt{\gamma / \beta}$ the number of localized modes is limited, and increases for increasing values of the imperfection/stiffness ratio. If $\gamma / \beta$ is very small no localized modes can occur. An evaluation of the limit value of the ratio for which at least the first symmetrical mode is localized yields $\gamma / \beta \geqslant 2 / \eta$. As a measure of the localization degree, the ratio $R$ between the elastic energy stored in the region of the defect and that relative to the whole system will be used in what follows.

The previous considerations lead to the conclusion that a minimum level of disorder must be present in the system in order to localize a given number of eigenfunctions. This statement is not in contrast with a well-known result from solid state physics according to which, "any disorder is sufficient to localize all the modes for arbitrary large coupling provided 
the system is taken to be large enough". Indeed, this last statement refers to a given random disorder extended to the whole chain, whereas the result found here concerns a finite section of disorder embedded in a larger regular system.

To make this concept clearer, let the length $l$ of the beam in Figure 2 tend to infinity. It is easy to show (see reference [23]) that in the limiting case $l=\infty$, the solution depends on the previously defined parameter $\gamma$ and the coupling parameter $\beta^{*}=E A /\left(k_{0} a^{2}\right)$, where $a$ is the extension of the defect. It is seen that even in the infinitely long beam a minimum value of the disorder to coupling ratio $\gamma / \beta^{*}$ is needed to localize modes higher than the first. However, this result concerns the case in which the extension of the defect $a$ is kept constant in the limit process. If $a$ is also made to vary (for instance by keeping the $a / l$ ratio constant, i.e., by fixing the level of disorder as a percentage fraction of the sum of substructure stiffnesses), $\beta^{*}$ can be rendered small anyway and it is thus possible that all modes up to the one desired become localized, as stated by solid state physics. Thus in this paper the expression "minimum disorder" must be understood to have the meaning indicated.

The problem analyzed is known in quantum mechanics as the problem of the rectangular potential well $[27,29]$. From a physical point of view, if the wave functions are localized, there is a high probability that the particle remains in the well; the localization degree $R$ previously defined corresponds to this probability. The deeper the well, the more energy levels there are, associated to motions confined to the well. On the contrary, if the wave functions are extended the particle may travel in either direction. However, unlike many quantum mechanics applications where an infinite domain is considered, here the motion is confined to the interval $[-1 / 2,1 / 2]$ and the energy spectrum is a discrete one; this is due to the boundary conditions that correspond to the presence of a barrier of potential of infinite height (potential box).

\subsection{PARAMETRIC ANALYSIS}

Some applications of results so far achieved can be illustrated as follows. In Figure 3 are shown the eigenfunctions of the system of Figure 2 when $\gamma=0 \cdot 1$ and $\beta$ assumes different values; if transition points are present, they are marked. In Figure 3(a) the first four vibration modes are illustrated for a beam with stiffness ratio $\beta=5 \times 10^{-4}$, for which only the first symmetrical mode is localized with degree $R=0.51$. Higher modes are of an extended type but suffer some distortion with respect to those of the perfect structure. In particular, in the third mode, the amplitude of oscillation in the region of the defect is considerably smaller than that relative to the external region. In addition, the associated frequencies are slightly different from those of the perfect system, $\lambda=1+n^{2} \pi^{2} \beta$.

When $\beta$ decreases the number of localized modes increases. In Figure 3(b) $\left(\beta=5 \times 10^{-5}\right)$ the first seven modal shapes and two higher modes are illustrated. The first two modes are localized with $R=0.92$ and 0.59 , respectively. Compared to the previous case, there is an increase in the localization degree of the first mode, together with a decrease in the frequency that approaches the lower limit value $\lambda=1-\gamma=0 \cdot 9$. Starting from $n=3$ the modes are extended, with distortions that decrease with increasing mode number. In the third mode the tendency just observed in the previous case is heightened and a marked reduction of the amplitude in the central region is observed. In this region there is one half-wave if the mode is symmetric and two half-waves if the mode is antisymmetric; when $n=27$ the symmetrical mode exhibits three half-waves in the central zone.

When $\beta=10^{-5}$ (see Figure 3(c)) the first four modes localize with ratios $R=0.99,0.95$, 0.86 and 0.51 respectively. The first frequency is $\lambda=0.907$ which is nearly equal to the limit value. 


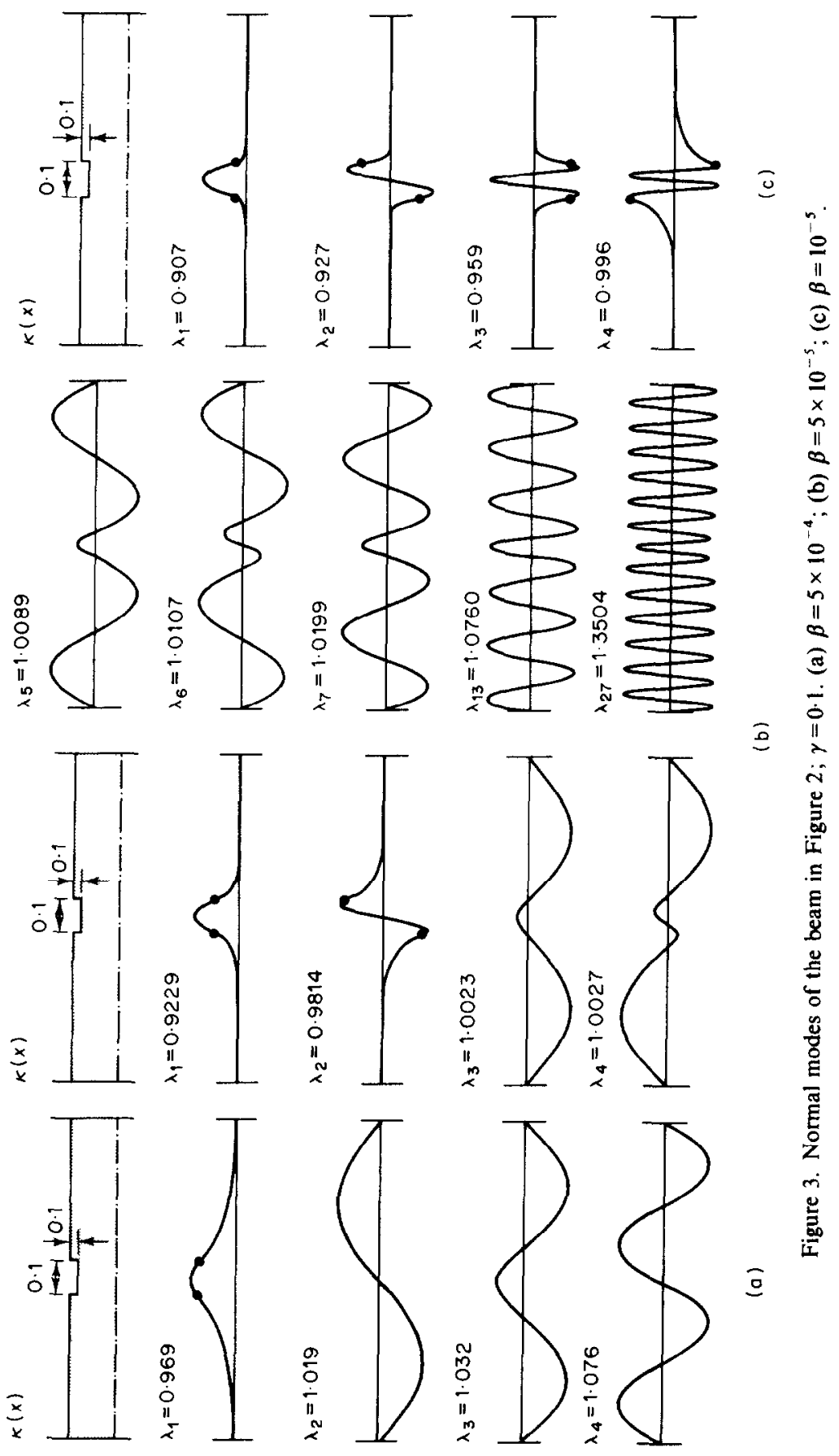




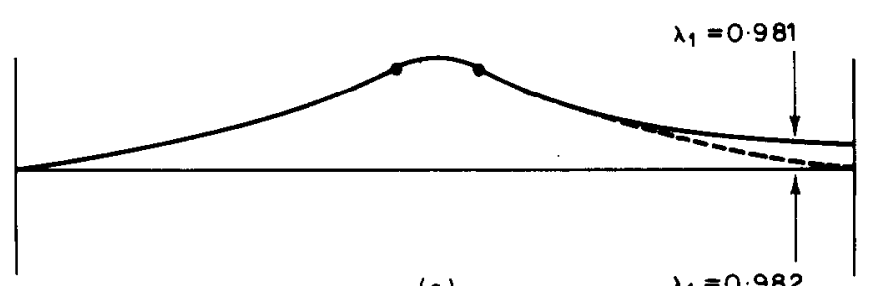

(a)

$\lambda_{1}=0.982$

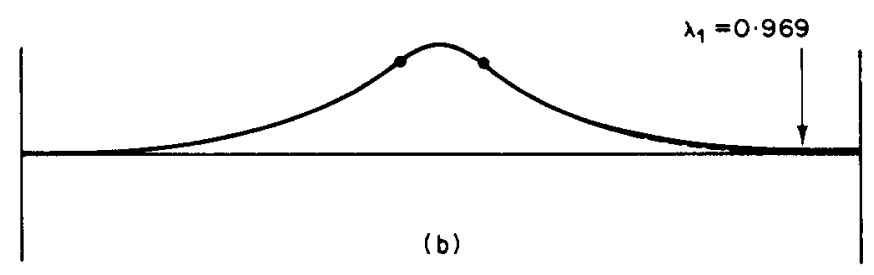

Figure 4. Localized modes for different boundary conditions: -_- , fixed-free; - - -, fixed-fixed; imperfection as in Figure 3. (a) $\beta=10^{-4}$; (b) $\beta=5 \times 10^{-4}$.

Let us now examine the influence of the boundary conditions. For a given imperfection, two different beams are considered, the previous one and a fixed-free beam. In Figure 4 are shown the eigenfunctions corresponding to two values of $\beta$ sufficient to localize only the first symmetrical mode. When $\beta=10^{-4}$ the frequencies are nearly equal but perceptible differences are present in the modal shape, which is more strongly localized when the beam is fixed at both ends. When $\beta=5 \times 10^{-4}$ frequencies and modes are practically indistinguishable; this is confirmed for decreasing values of $\beta$. In conclusion, the influence of the boundary conditions, which is small on the frequencies anyway, is noticeable on the modal shapes only if the modes are weakly localized. In the opposite case the decay of the eigenfunction is such that the solution is independent of the boundary conditions. Under these conditions the modal shapes can be determined by referring to the model of an infinitely long beam.

To confirm this result let us again consider the fixed-free beam and vary the position of the defect. For a fixed value $\beta=5 \times 10^{-5}$ the four cases illustrated in Figure 5 have been analyzed. With $\xi$ denoting the mid-point of the defect, it is seen that when $\xi=0.25,0.75$ the frequencies do not change, while the modal shapes coincide to within a shift. Frequencies and modes are also identical with the first two of Figure 3(b), relative to the fixedfixed beam with the defect located at mid-span $(\xi=0.5)$. If the defect is very near the supports, i.e., if $\xi=0.1$ or 0.9 , there are small variations in the frequencies and noticeable changes in the modal shapes.

\section{DIFFUSED IMPERFECTIONS}

Diffused imperfections distributed along the beam axis will now be considered. A very special type of imperfection, namely a periodic (or nearly periodic) imperfection, will be dealt with first, and then non-periodic disorder will be analyzed.

\subsection{PERIODIC AND NEARLY PERIODIC IMPERFECTIONS}

Let the function $\chi(x)$ in equation (4) be periodic with period $d, \chi(x+d)=\chi(x)$. Then equation (2) is a homogeneous Hill equation to which the Floquet theory of periodic differential equations applies. It is well known that the solution of such an equation can be easily found for any $x$ if the solution is known over one period of the coefficients (the 


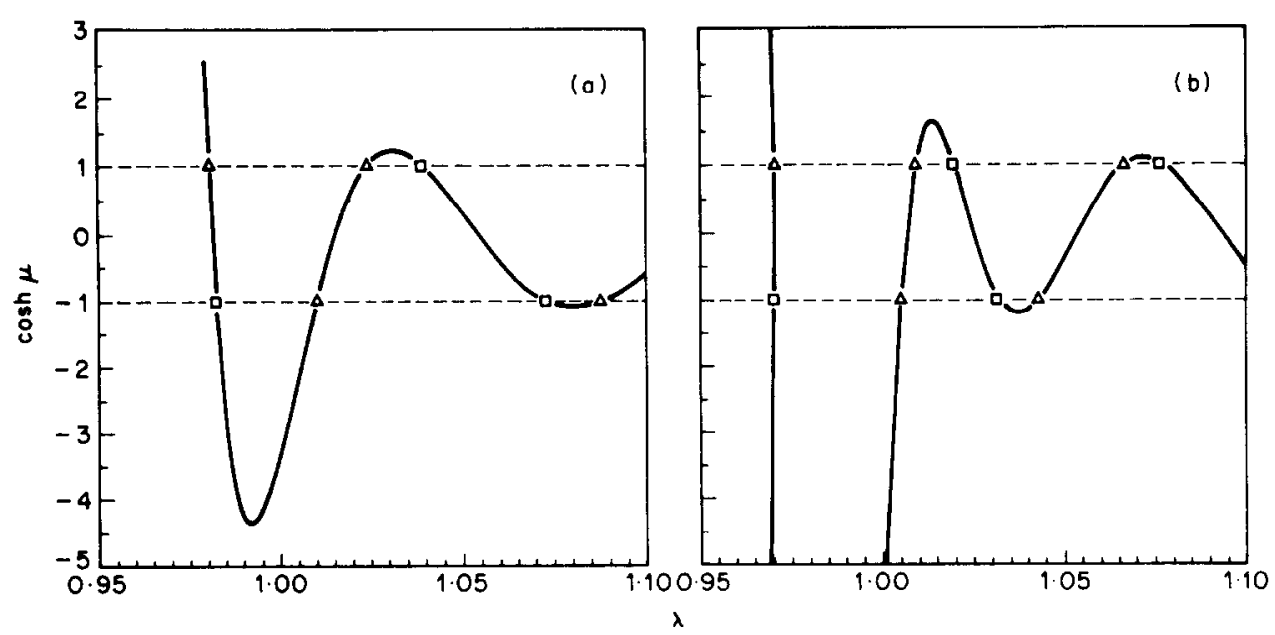

Figure 6. Pass- and stop-bands for the element in Figure $2 ; \gamma=0.1, \eta=0.05$. (a) $\beta=10^{-3}$; (b) $\beta=5 \times 10^{-4}$. $\triangle$, Free-free frequencies; $\square$, fixed-fixed frequencies.

and horizontal lines $\cosh \mu= \pm 1$, projected onto the $\lambda$-axis, delimit the pass-bands. The first two bounding frequencies are found to be unlocked (free-free) and locked (fixedfixed) frequencies, respectively; then, pairs of unlocked and locked frequencies alternate in the range examined, differently from certain uniform elements [32]. For a fixed $\beta$ the pass-band width increases with $\lambda$, but the pass-bands shrink and move to the left when $\beta$ decreases, thus entailing higher modal density. For example, the first pass-band is bounded by frequencies of 0.9801 and 0.9822 when $\beta=10^{-3}$ and frequencies of 0.96915 and 0.96926 when $\beta=5 \times 10^{-4}$. This is in accordance with the previous remarks about the little influence exerted by boundary conditions when $\beta$ is small enough. The two values of $\beta$ considered are really not very small (compare them with those in Figure 3 ) so that only the first passband lies on the left side of $\lambda=1$ : i.e., only the first mode exhibits turning points.

Let us now consider a four-bay chain $(N=4)$ fixed at the ends. There are three frequencies in any pass-band to which locked frequencies have to be added. In the first pass-band they are $0.9804,0.9811,0.9818$ and $0.96917,0.96921,0.96924$ for the two values of $\beta$, respectively. Note that, due to the narrowness of the propagation zone, frequencies are nearly degenerate. The associated modal shapes are plotted in Figure 7 together with the locked modes. It can be seen that even if the modes are localized within the single elements, where two turning points are present, the modes are extended to the whole. In fact, displacements repeat themselves in any bay, simply being modulated by a sinusoidal law. When $\beta$ decreases, localization increases in the single unit, but the repetitive character of the modes remains unchanged. For very small values of $\beta$ the modes become trains of impulses.

From a theoretical point of view such modes have to be considered to be extended, so that there is transmission of energy along the beam, provided that the frequencies lie in the pass-band. However, since the pass-band width rapidly tends to zero, there is transmission only if resonant conditions occur. Therefore, from a practical point of view, the beam behaves as a system the modes of which are all localized in a given frequency range: if a random frequency force is acting at one end of the beam, the averaged response at the other end is nearly zero, except for a few, anomalous events in which the driving frequency lies in one of the vanishingly small pass-bands.

This result refers to the special case of periodic imperfections. However, such imperfections represent an ideal case, because a periodic structure is, in one sense, still a perfect 

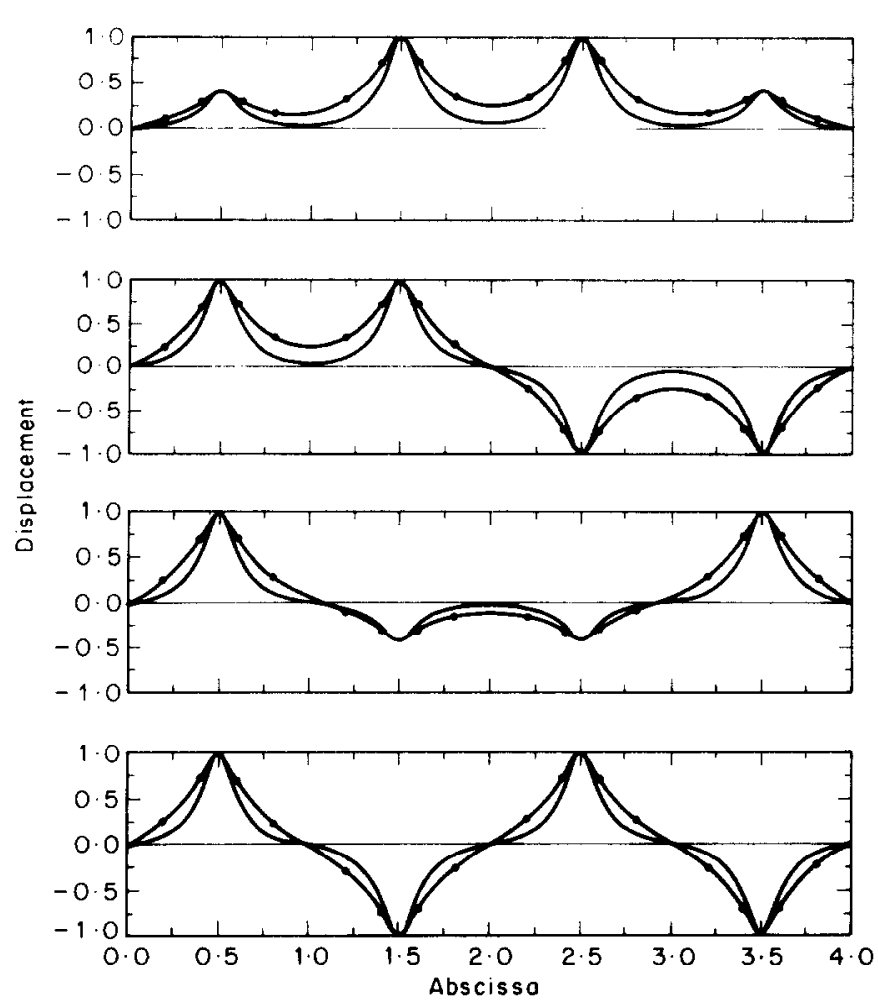

Figure 7. Natural modes for a four-bay periodic chain: imperfections as in Figure $6 ;-\bullet, \beta=10^{-3} ;-$, $\beta=5 \times 10^{-4}$.

system. Thus it is interesting to investigate the dynamical behaviour of the beam when a small deviation from perfect periodicity occurs: i.e., when the system is affected by nearly periodic imperfections.

To this end let one or more elements of the chain have mechanical properties that are slightly different from the other, identical, elements. The system frequencies are then modified by small quantities, and some of them can leave the standard pass-band. If this occurs, the corresponding modal shapes are exponentially modulated in the periodic section of the system, so they localize near the anomalous element. This behaviour has already been illustrated in reference [4] for the weak localization case.

Still with reference to the four-bay system, it is assumed here that the second element from the left is affected by imperfections of different amplitude, compared to the remaining elements. The system transfer matrix can thus be evaluated as $T^{2} T^{\prime} T, T^{\prime}$ being the matrix of the special sub-element, and the characteristic equation can be obtained by making the $(1,2)$ element equal to zero. Successively, the modal shape is obtained by repeated matrix multiplications of the state vector by the relevant transfer matrix.

In Figure 8 are shown the first four modes obtained when $\beta=10^{-3}$ and $\eta=0.05$ for all elements, while $\gamma=0.1$ for the standard ones and $\gamma^{\prime}=0.095$ or $\gamma^{\prime}=0.080$ for the special element. Curves should be compared with those in Figure 7 corresponding to the same $\beta$, concerning the periodic case. A tendency towards localization is observed, the tendency becoming more marked when deviations from perfect periodicity increase. The modal shape characteristics can be better understood if the associated frequencies are compared with the position of the pass-bands of the two element types. When $\gamma^{\prime}=0.095$ the bounding 

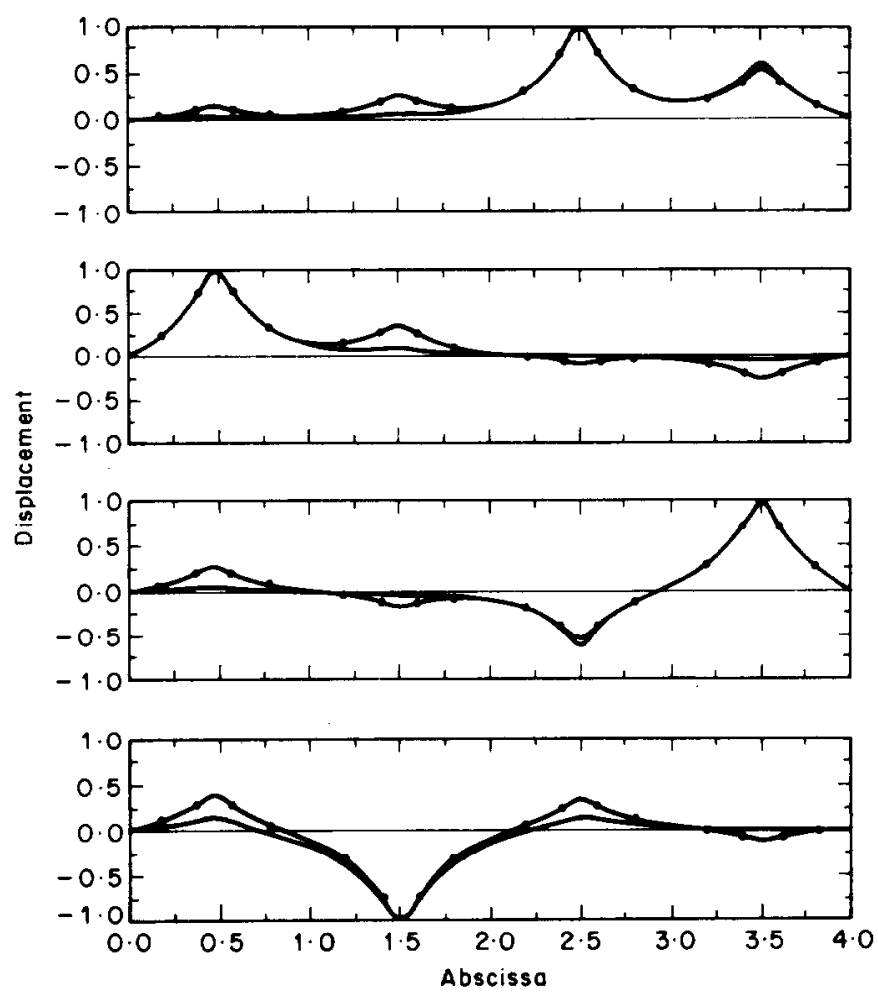

Figure 8. Natural modes for a four-bay nearly periodic chain; $\beta=10^{-3}, \eta=0.05 ; \gamma=0.1$ for the first, third and fourth element; -,$- \gamma^{\prime}=0.095 ; \_, \gamma^{\prime}=0.08$ for the second element.

frequencies of the first pass-band are found to be $\lambda=0.9817,0.9839$ (it should be remembered that the corresponding values for the standard pass-band are 0.9801 and 0.9822 ) so that the two pass-bands overlap. Two system frequencies are found to lie in the standard pass-band, the third one in the common zone, and the fourth in the pass-band of the special element. When $\gamma^{\prime}=0.080$ the bounding frequencies become $\lambda=0.9860$ and 0.9890 and the pass-bands do not overlap. In this case three frequencies lie in one pass-band and the fourth frequency in the other. This explains the modifications of the modal shapes in passing from the periodic to the nearly periodic case: they are due to the decay of the oscillation amplitude in the anomalous element in the first three modes and in the standard elements in the fourth.

Note that the range encompassing the first four frequencies expands when periodicity is broken. On the one hand, this fact increases the probability of having a non-zero response far removed from the application point of a random force but, on the other, it obstructs the transmission of energy through the chain, since modes tend to localize.

The example illustrated concerns a nearly periodic imperfection; from previous considerations it is expected that, when disorder is of non-periodic type, the tendency already manifested becomes more marked. This subject is discussed in the next subsection.

\subsection{NON-PERIODIC IMPERFECTIONS}

Let us now consider the imperfection $\chi(x)$ to be non-periodic. A numerical analysis is performed here referring to a beam with a fixed end (at $x=0$ ) and a free end (at $x=1$ ), 
by considering exponentially modulated imperfections

$$
\chi(x)= \pm \mathrm{e}^{\varphi x} \sin (r \pi x / 2),
$$

where $\varphi$ and $r$ are constants. Frequencies and modes of vibration are determined by applying the Galerkin technique with the sinusoidal eigenfunctions of the perfect system taken as shape functions.

The first example treated (see Figure 9) reveals the effect of the interaction between several stiffness gaps. The stiffness of the springs presents two gaps, the $\gamma / \beta$ ratio being quite small. The first eigenfunction is shown at the bottom of the figure; it displays four transition points corresponding to the intersections of the horizontal line $\lambda=0.987$ (obtained numerically) with the curve $\kappa(x)$. In these points the character of the solution changes from oscillating to exponential or vice versa. In the case concerned, due to the closeness of the two gaps and the small value of the $\gamma / \beta$ ratio, the mode does not localize; however, the tendency to localize at the two gaps is noticed.

The localization takes place when $\beta$ decreases, $\gamma$ being held constant. In Figure 10 are shown the first five modes when $\beta$ is very small, and so all these modes are localized, since $\lambda<1$. It is noted that the first and third modes, as well as the fourth and fifth modes, are similar to within a shift, if the symmetry conditions are accounted for. However, due to the different depths of the gaps, the frequencies are no longer nearly degenerate as in the case of periodic imperfections, and the band character of the frequencies is lost. It should be noted that a higher degree of localization is associated with the modes localized near the deepest gap, since closer turning points are associated with lower frequencies.

In conclusion, the results given confirm that if the value of the $\gamma / \beta$ ratio is sufficient to localize the modes near the individual gaps there is no interaction between them. This suggests the possibility of making a separate study of the effect of each stiffness gap, by treating it as an isolated defect, thus entailing a marked reduction in the computational difficulties. The problem will be analysed in the next section.

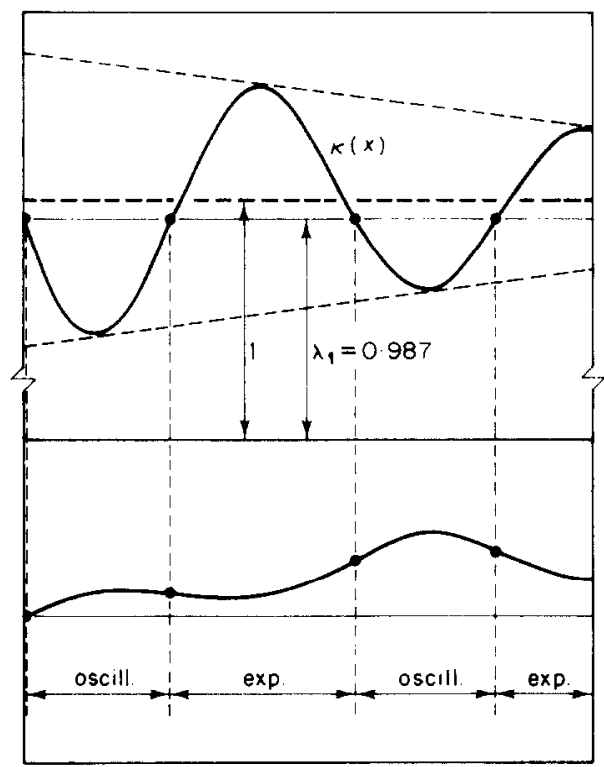

Figure 9. Diffused imperfections and turning points; $\beta=10^{-3}, \gamma=0 \cdot 1, \varphi=-0 \cdot 7, r=7$. 
By applying a standard technique (see reference [30]), the functions $\phi_{0}(x)$ and $\phi_{1}(x)$ are obtained by integrating the appropriate perturbation equations: the following solution is thus derived:

$$
\begin{gathered}
u_{1}(x)=[1 / \sqrt[4]{\lambda-\kappa(x)}]\left[a_{1} \cos \psi_{1}(x)+b_{1} \sin \psi_{1}(x)\right], \\
u_{2}(x)=[1 / \sqrt[4]{\kappa(x)-\lambda}]\left\{a_{2} \exp \psi_{2}(x)+b_{2} \exp \left[-\psi_{2}(x)\right]\right\}
\end{gathered}
$$

where

$$
\psi_{1}(x)=\beta^{-1 / 2} \int_{x}^{\Delta(x)} \sqrt{\lambda-\kappa(x)} \mathrm{d} x, \quad \psi_{2}(x)=\beta^{-1 / 2} \int_{\Delta(\lambda)}^{x} \sqrt{\kappa(x)-\lambda} \mathrm{d} x .
$$

Equations (10) are singular at the transition point so that they are not uniformly valid. However, they are a good approximation of the exact solution except in the neighbourhood of $x=\Delta$. For this reason they are called outer solutions and are denoted by $u_{o}(x)$. The basic idea of the WKB method is to determine a third solution valid in this region (inner solution $\left.u_{i}(x)\right)$ and then to match the three asymptotic expansions.

Near the point $x=\Delta$, the function $\lambda-\kappa(x)$ can be approximated by a first order Taylor expansion, so that equation (2) can be replaced by

$$
\beta u^{\prime \prime}+(\Delta-\kappa) \kappa^{\prime}(\Delta) u=0, \quad \text { or } \quad \mathrm{d}^{2} u / \mathrm{d} z^{2}-z u=0,
$$

if the following change of variable is introduced:

$$
z=\beta^{-1 / 3}\left[\kappa^{\prime}(\Delta)\right]^{1 / 3}(x-\Delta) .
$$

Equation (13) is Airy's equation, the general solution of which is

$$
u_{3}(z)=a_{3} \mathrm{Ai}(z)+b_{3} \mathrm{Bi}(z),
$$

$\mathrm{Ai}(z)$ and $\mathrm{Bi}(z)$ being Airy's functions of first and second type, respectively [36].

In order to describe the solution properly in the whole interval $[0, \infty)$ it is necessary to match the $u_{o}$ and $u_{i}$ solutions, by suitably selecting four of the six arbitrary constants. The operation is performed by applying the principle of matching which states that the inner limit of the outer solution must be equal to the outer limit of the inner solution. In symbols, $\left(u_{o}\right)_{i}=\left(u_{i}\right)_{o}$. By making the series expansion of equations (10) for small $|x-\Delta|$ equal to the expansion of equation (15) for large $z$ (i.e., for $|x-\Delta|>O\left(\beta^{1 / 3}\right)$ ) it follows that

$$
\begin{gathered}
u_{1}(x)=[1 / \sqrt[4]{\lambda-\kappa(x)}]\left\{c_{2} \cos \left[\psi_{1}(x)+\pi / 4\right]+c_{1} \sin \left[\psi_{1}(x)+\pi / 4\right]\right\}, \\
u_{2}(x)=[1 / \sqrt[4]{\kappa(x)-\lambda}]\left\{c_{2} \exp \psi_{2}(x)+\frac{1}{2} c_{1} \exp \left[-\psi_{2}(x)\right]\right\}, \\
u_{3}(z)=\frac{\sqrt{\pi}}{\left[\beta^{1 / 2} \kappa^{\prime}(\Delta)\right]^{1 / 6}}\left[c_{1} \operatorname{Ai}(z)+c_{2} \operatorname{Bi}(z)\right],
\end{gathered}
$$

where asymptotic properties of Airy's functions have been taken into account. Equation (16) is defined to within only two arbitrary constants $c_{1}$ and $c_{2}$.

The three different branches of the solution can be combined to obtain a unique expression valid on the whole real axis, called the composite solution:

$$
u_{c}=u_{o}+u_{i}-\left(u_{o}\right)_{i}=u_{o}+u_{i}-\left(u_{i}\right)_{o} .
$$

Indeed, since $\left(u_{i}\right)_{o} \approx u_{i}$ in the outer region and $\left(u_{o}\right)_{i} \approx u_{o}$ in the inner region, it ensues that $u_{\mathrm{c}} \approx u_{o}$ outside and $u_{c} \approx u_{i}$ inside the region of non-uniformity.

Let us now consider the boundary conditions. $u_{2}(\infty)=0$ entails $c_{2}=0$; by imposing $u_{1}^{\prime}(0)=0$ (symmetrical modes) or $u_{1}(0)=0$ (antisymmetrical modes) and remembering that 
$\kappa^{\prime}(0)=0$ (symmetrical imperfection), $\psi_{1}(0)=\pi(n-1 / 2) / 2$ is obtained and therefore, from equation (11a), we obtain

$$
\int_{0}^{\Delta(\lambda)} \sqrt{\lambda-\kappa(x)} \mathrm{d} x=\frac{\pi}{2}\left(n-\frac{1}{2}\right) \beta^{1 / 2} \quad(n=1,2, \ldots) .
$$

Equation (18) permits evaluation of the frequency of oscillation $\lambda$ of the localized mode.

It is worthwhile at this point commenting on the procedure adopted and the limits of validity of the solution obtained. Above all, it is observed that the condition at $x=0$ was imposed on the function $u_{1}(x)$ : i.e., on the external solution. It was implicitly admitted that the origin does not belong to the region of non-uniformity; this is true only if the transition point is sufficiently far from $x=0$; i.e., if $\Delta>O\left(\beta^{1 / 3}\right)$. Since the abscissa of the turning point increases for increasing eigenvalues, $\kappa(x)$ being monotonic, the accuracy of the solution improves at higher modes. As a second comment it is noted that the nonuniformity region shrinks for increasing $n$. Indeed, larger $\kappa^{\prime}(\Delta)$ correspond to larger $\Delta$ and, therefore, because of equation (14), values of $|z|$ increase more rapidly with the distance from the turning point. This circumstance entails a more rapid tendency of Airy's functions to assume periodic or exponential behaviour. This is in accordance with the fact that the more rapidly $|\lambda-\kappa(x)|$ increases in the neighbourhood of the zero of the function, the more rapidly the transitory behaviour decays. This consideration also shows that the method furnishes results the accuracy of which improves with mode number.

\subsection{ILLUSTRATIVE PROBLEM}

As an application of the method let us consider a parabolic stiffness gap,

$$
\kappa(x)=1-\gamma\left(1-x^{2}\right),
$$

where $\gamma$ is the maximum amplitude of the imperfection. This problem corresponds to the one-dimensional harmonic oscillator problem in quantum mechanics [27-29] in which, however, equation (19) holds good in the whole interval $[0, \infty)$ instead of in $[0,1]$.

If $1-\gamma<\lambda<1$ the equation $\lambda-\kappa(x)=0$ admits the solution $x=\Delta$ (turning point) with

$$
\Delta=\sqrt{(\lambda-1+\gamma) / \gamma} .
$$

By replacing $\kappa(x)$ and $\Delta$ (equations (19) and (20)) in equation (18), integrating and solving with respect to $\lambda$ we obtain

$$
\lambda=1-\gamma+\sqrt{\beta \gamma}(2 n-1), \quad n=1,2, \ldots, n_{0},
$$

and then, from equation (20),

$$
\Delta=(\beta / \gamma)^{1 / 4} \sqrt{2 n-1}, \quad n=1,2, \ldots, n_{0} .
$$

In equations (21) and (22) $n_{0}$ is the number of localized modes, evaluated by imposing $\lambda<1$ :

$$
n_{0}<\frac{1}{2}(1+\sqrt{\gamma / \beta}) .
$$

The largest integer that satisfies the inequality is the number of localized modes; so the higher the ratio $\gamma / \beta$, the greater the number of modes that localize. From equations (21)(23) the following conclusions can be drawn about the influence of the shape of the imperfection: other conditions being equal, for increasing amplitude $\gamma$ of the defect, or for decreasing size $a$, the transition point $x=a \Delta$ (in dimensional variables) approaches the origin, heightening the localized character of the mode; however, both effects can reduce the number of localized modes. 
The eigenfunctions associated with the eigenvalues (21) are obtained from equations (16) and (11), it being recalled that $c_{2}=0$. We get

$$
\begin{gathered}
u_{1}(x)=\sin \left\{\psi_{1}(x)+\pi / 4\right\} / \sqrt[4]{1-x^{2} / \Delta^{2}}, \quad u_{2}(x)=\frac{1}{2} \exp \left\{-\psi_{2}(x)\right\} / \sqrt[4]{1-x^{2} / \Lambda^{2}} . \\
u_{3}(z)=\sqrt{\pi}(n-1 / 2)^{1 \cdot \hbar} \text { Ai }(z)
\end{gathered}
$$

where

$$
\begin{gathered}
\psi_{1}(x)=\left(n-\frac{1}{2}\right)\left[(\pi / 2)-\arcsin (x / \Delta)-(x / \Delta) \sqrt{1-x^{2} / \Delta^{2}}\right], \quad 0 \leqslant x<\Delta, \\
\psi_{2}(x)=\left(n-\frac{1}{2}\right)\left[(x / \Delta) \sqrt{\left(x^{2} / \Delta^{2}\right)-1}-\ln \left\{(x / \Delta)+\sqrt{\left(x^{2} / \Delta^{2}\right)-1}\right\}\right], \quad \Delta<x \leqslant 1, \\
\psi_{2}(x)=\psi_{2}(1)+\sqrt{(1-\lambda) / \beta}(x-1), \quad x \geqslant 1,
\end{gathered}
$$

and

$$
z=\sqrt[3]{2(2 n-1)^{2}}[(x / \Delta)-1] .
$$

The functions $u_{1}(x), u_{2}(x)$ and $u_{3}(x)$ show a marked property of the modes of vibration: if the ratio $x / \Delta$ is assumed to be an independent variable, the eigenfunctions do not depend on the parameters $\beta$ and $\gamma$ in the interval $0 \leqslant x / \Delta<1$. Since $\Delta=\Delta(\beta / \gamma)$ the modal shapes depend only on the ratio between the two parameters. This property holds good outside the defect too, as is shown by equations (25c) and (21).

The first three localized eigenfunctions are illustrated in Figure $11 \mathrm{vs}$. the $x / \Delta$ ratio. The two branches (equations $(24 \mathrm{a}, \mathrm{b})$ ) of the outer solution $u_{o}$, the inner solution $u_{i}$ (equation (24c)) and the composite solution $u_{c}$ (equation (17)) are shown. The outer solution has been drawn by taking equation $(25 b)$ to be valid: i.e., $x \leqslant 1$ is assumed. The curves confirm that the extension of the region of non-uniformity of the outer solution decreases for increasing mode number $n$. This is highlighted by the fact that, on passing to higher modes, the interval in which the composite solution moves away from the inner and outer solutions decreases.

\section{CONCLUSIONS}

Free longitudinal oscillations of a beam with small axial stiffness, continuously restrained by elastic springs, have been studied. With account taken of small imperfections in the stiffness of the springs the localization phenomenon of the modal shapes has been analyzed. The following conclusions have been drawn.

(1) The phenomenon can be tackled as a classical problem of mathematical physics, called the turning point problem. In fact, the eigenfunctions display transition points in which the character of the solution changes from oscillating to exponential or vice versa, so that the amplitude of the displacement is magnified in one or more regions of the beam of limited size. Analogies with similar problems in quantum mechanics have been emphasized.

(2) If a finite section of disorder is embedded in a larger regular system, the localization occurs when the ratio between the amplitude of the imperfection and the coupling comes to have sufficiently high values. Therefore, localization is promoted by high modal densities.

(3) Only the first few modes of vibrations are localized and their number increases as the imperfection/stiffness ratio increases.

(4) The degree of localization of a mode increases as the extension of the defect decreases and its amplitude increases. 

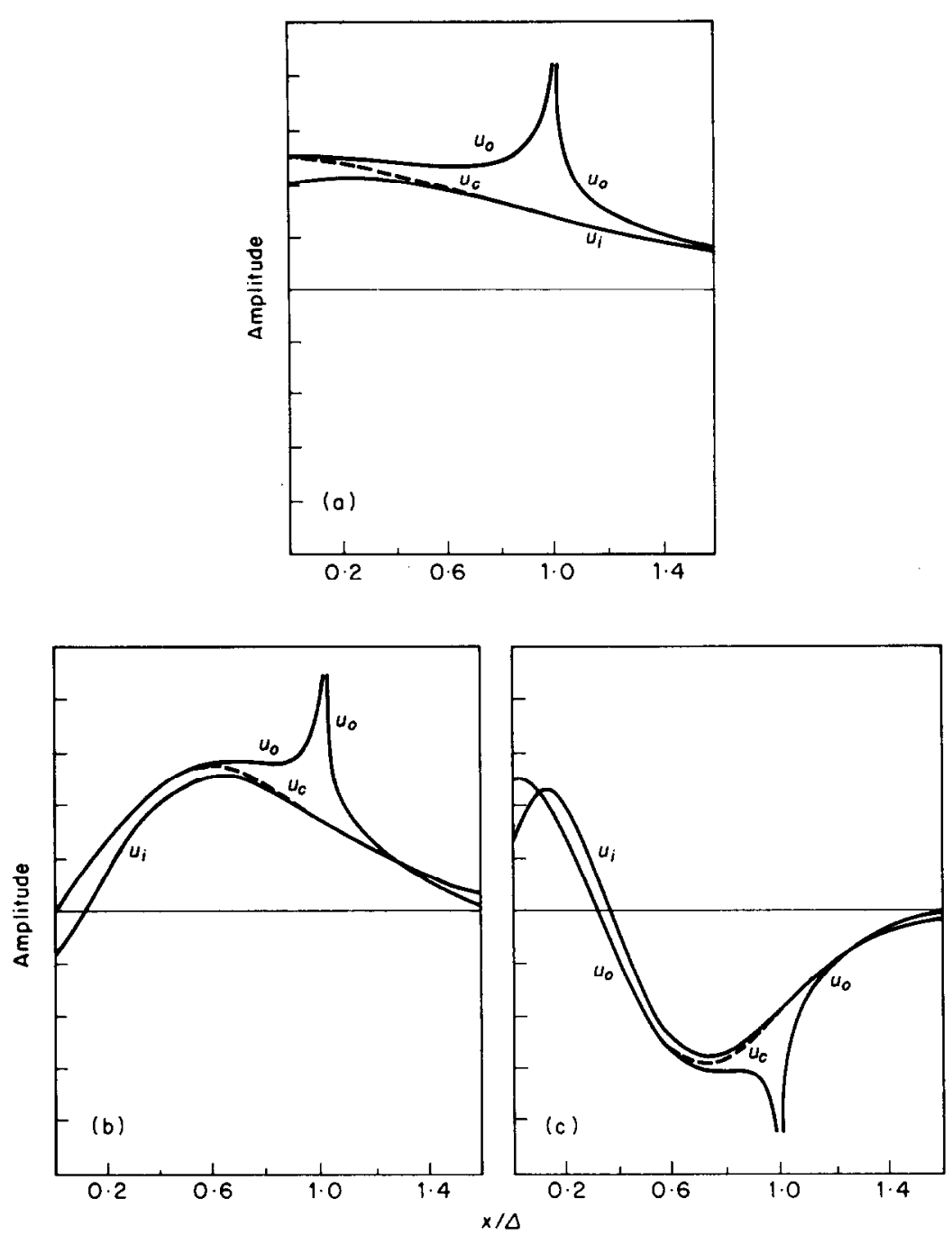

Figure 11. Localized modes of a beam of infinite length with a parabolic defect of stiffness. (a) $n=1$; (b) $n=2$; (c) $n=3$.

(5) If the imperfection is localized, modal shapes localize around the imperfection. If the decay of the eigenfunction is sufficiently fast, the mode and the frequency are independent of boundary conditions and length of the beam, so they can be evaluated by using the infinite beam model.

(6) If the imperfection is periodically diffused, a pass-band behaviour occurs. If the disorder to coupling ratio is sufficiently large, normal modes are trains of impulses extended to the whole beam. However, the pass-band width in which frequencies are contained is so narrow that only resonant travelling waves can be transmitted along the beam. In this condition even small deviations from pure periodicity are sufficient to destroy the passband behaviour and to localize the modes.

(7) If the imperfection is of a diffused type but the stiffness gaps are sufficiently far away from each other the modes localize near the individual gaps, as if they were isolated. In this case it is possible to perform a local analysis by referring to the model of a beam of infinite length with a unique stiffness gap. 
(8) The asymptotic WKB method is an efficient tool for evaluating the localized eigenfunctions and the associated eigenvalues. An example concerning a parabolic imperfection has been dealt with in detail.

\section{REFERENCES}

1. P. W. ANDERSON 1958 Physical Review 109, 1492-1505. Absence of diffusion in certain random lattices.

2. D. C. Herbert and R. Jones 1971 Journal of Physics C: Solid State Physics 4, 1145-1161. Localized states in disordered systems.

3. P. Erdös and R. C. Herndon 1982 Advances in Physics 31(2), 65 163. Theories of electrons in one-dimensional disordered systems.

4. A. J. Keane and W. G. Price 1989 Journal of Sound and Vibration 128, 423-450. On the vibrations of mono-coupled periodic and near-periodic structures.

5. C. PierRe 1990 Journal of Sound and Vibration 139, 111-132. Weak and strong vibration localization in disordered structures: a statistical investigation.

6. C. Pier Re and E. H. Dowell 1987 Journal of Sound and Vibration 114. 549-564. Localization of vibrations by structural irregularity.

7. C. PIER RE and P. D. ChA 1989 American Institute of Aeronautics and Astronautics Journal 27(2), 227-241. Strong mode localization in nearly periodic disordered structures.

8. G. J. KISSEL 1987 AIAA Paper 87-0819, Proceedings of the 28th Structures, Structural Dynamics and Material Conference, Monterey, California. Localization in disordered periodic structures.

9. G. J. Kissel 1988 Ph.D. Dissertation, Massachussets Institute of Technology. Localization in disordered periodic structures.

10. W. T. KoITER 1967 NASA Technical Report F10-833. On the stability of elastic equilibrium.

11. O. O. BENDIKSEN 1987 American Institute of Aeronautics and Astronautics Journal 25(9), 1241-1248. Mode localization phenomena in large space structures.

12. C. Pierre, D. M. TANG and E. H. Dowell 1987 American Institute of Aeronautics and Astronautics Journal 25(9), 1249-1257. Localized vibrations of disordered multispan beams: theory and experiment.

13. C. Pierre 1988 Journal of Sound and Vibration 126, 485-502. Mode localization and eigenvalue loci veering phenomena in disordered structures.

14. C. H. Hodges and J. Woodhouse 1983 Journal of the Acoustical Society of America 79(9), 894-905. Vibration isolation from irregularity in a nearly periodic structure: theory and measurements.

15. C. PIERRE 1989 International Journal of Control 49(2), 521-532. Root sensitivity to parameter uncertainties: a statistical approach.

16. R. A. IbRAhim 1987 Applied Mechanics Reviews 40(3), 309-328. Structural dynamics with parameter uncertainties.

17. C. H. Hodges 1982 Journal of Sound and Vibration 82, 411-424. Confinement of vibration by structural irregularity.

18. C. H. Hodges and J. Woodhouse 1989 Journal of Sound and Vibration 130, 253-268. Confinement of vibration by one-dimensional disorder, II : a numerical experiment on different ensemble averages.

19. S. T. Wei and C. Pierre 1989 Journal of Sound and Vibration 129, 397-416. Effects of dry friction damping on the occurrence of localized forced vibrations in nearly cyclic structures.

20. P. J. CORNWELL and O. BENDIKSEN 1989 American Institute of Aeronautics and Astronautics Journal 27(2), 219-226. Localization of vibrations in large space reflectors.

21. C. H. Hodges and J. Woodhouse 1989 Journal of Sound and Vibration 130, 237-251. Confinement of vibration by one-dimensional disorder, $\mathrm{I}$ : theory of ensemble averaging.

22. J. F. M. SCOTT 1985 Proceedings of the Royal Society of London A398, 341-363. The statistics of waves propagating in a one-dimensional random medium.

23. A. Luongo 1988 Proceedings of the IX Italian Congress of Theoretical and Applied Mechanics, Bari 2,655-658. Localized vibrations in imperfect structures with high modal density (in Italian).

24. A. LuONGo and M. Pignataro 1988 American Institute of Aeronautics and Astronautics Journal 26(11), 1395-1402. Multiple interaction and localization phenomena in the postbuckling of compressed thin-walled members.

25. A. Luongo 1991 International Journal of Solids and Structures 27(15), 1943-1954. On the amplitude modulation and localization phenomena in interactive buckling problems. 
26. C. Pierre 1989 Journal of Applied Mathematics and Physics (ZAMP) 40,758-761. Curve veering and mode localization in a buckling problem.

27. L. D. LANDAu and E. M. Lifchitz 1966 Quantum Mechanics, Non-relativistic Theory (in French). Moscow: Mir.

28. R. McWeEny 1972 Quantum Mechanics: Principles and Formalism. Oxford: Pergamon Press.

29. A. A. Sokolov, I. M. Ternov and V. Ch. Zhukovskir 1984 Quantum Mechanics. Moscow: Mir.

30. A. NAYFEH 1973 Perturbation Methods. New York: John Wiley.

31. J. A. Richards 1983 Analysis of Periodically Time-varying Systems. Berlin: Springer-Verlag.

32. G. SEN GUPTA 1970 Journal of Sound and Vibration 13, 89-101. Natural flexural waves and the normal modes of periodically-supported beams and plates.

33. D. J. MEAD 1970 Journal of Sound and Vibration 11, 181-197. Free wave propagation in periodically supported, infinite beams.

34. D. J. MEAD 1975 Journal of Sound and Vibration 40, 1-18. Wave propagation and natural modes in periodic systems, $\mathbf{I}$ : mono-coupled systems.

35. M. G. FAulkner and D. P. Hong 1985 Journal of Sound and Vibration 99, 29-42. Free vibrations of a mono-coupled periodic system.

36. M. Abramowitz and A. Stegun (editors) 1972 Handbook of Mathematical Functions. New York: Dover.

\section{APPENDIX A: DYNAMICS OF PERIODIC BEAMS}

The following is a brief outline of the theory of periodic beams.

Let the length of the beam be a multiple of the period $d$, say $l=N d$. Then, from a mechanical point of view, the system can be considered as a chain on $N$ identical subsystems. The dynamic flexibility matrix relates displacements $u$ and internal forces $f=\beta u^{\prime}$ at the left and right ends of the $k$ th element, namely

$$
\left\{\begin{array}{l}
u_{L} \\
u_{R}
\end{array}\right\}=\left[\begin{array}{ll}
\alpha_{L L} & \alpha_{L R} \\
\alpha_{R L} & \alpha_{R R}
\end{array}\right]\left\{\begin{array}{l}
f_{L} \\
f_{R}
\end{array}\right\}
$$

where $\alpha_{L L}$ and $\alpha_{R R}$ are direct receptances and $\alpha_{L R}=\alpha_{R L}$ is the cross-receptance, all quantities depending on the frequency $\lambda$. By imposing continuity and equilibrium conditions between the $k$ th and $(k+1)$ th elements, i.e., $u_{R, k}=u_{L, k+1}$ and $f_{R, k}=-f_{L, k+1}$, the following relation is obtained:

$$
\left\{\begin{array}{l}
u_{L} \\
f_{L}
\end{array}\right\}_{k+1}=\frac{1}{\alpha_{L R}}\left[\begin{array}{cc}
\alpha_{R R} & \alpha_{L R}^{2}-\alpha_{L L} \alpha_{R R} \\
-1 & \alpha_{L L}
\end{array}\right]\left\{\begin{array}{l}
u_{L} \\
f_{L}
\end{array}\right\}
$$

This defines the $2 \times 2$ transfer matrix $T=\left[t_{i j}\right]$. The eigenvalues $\sigma_{j}(j=1,2)$ of matrix $T$ are the characteristic multipliers, the meaning of which follows from the Floquet theorem: two linearly independent solutions to the differential equations exist in which the state vector is altered only by the complex constant $\sigma_{j}$ when it is evaluated at two different points one period $d$ apart. Since det $T=1, \sigma_{1} \sigma_{2}=1$ and $\sigma_{1}+\sigma_{2}=\operatorname{tr} T$. By defining $\sigma_{1,2}=$ $\exp ( \pm \mu), 2 \cosh \mu=$ tr $T$ follows. The parameter $\mu$ (proportional to the Floquet characteristic exponent $\rho, \mu=\rho d$ ) is called the propagation constant, and is a function of the frequency $\lambda$ via the relationship

$$
\cosh \mu=\left(\alpha_{L L}+\alpha_{R R}\right) / 2 \alpha_{L R} .
$$

The real part of $\mu, \operatorname{Re}(\mu)$, represents the logarithmic decay of the amplitude solution, and the imaginary part, $\operatorname{Im}(\mu)$, the phase difference in two adjacent elements. Wave propagation can only exist provided that $\mu$ is imaginary, and this occurs when $|\cosh \mu| \leqslant 1$. 
The real and imaginary values of $\mu$ furnished by equation (A 3 ) give rise to pass- and stopbands within the frequency spectrum inside which the transmission of energy is allowed or forbidden.

It should be noted that in the particular case in which the element is symmetric about its centre $\left(\alpha_{L L}=\alpha_{R R}\right.$, i.e., $\left.t_{11}=t_{22}\right)$, the natural frequencies of the fixed-fixed (locked) and free-free (unlocked) individual subsystem, obtained by posing $t_{12}=0$ or $t_{21}=0$ respectively, lie at the boundaries of the propagation zones. In fact, det $T=1$ entails $\operatorname{tr} T= \pm 2$, and so $\cosh \mu= \pm 1$. If the unlocked and locked natural frequencies alternate, then any propagation zone is delimited by a free frequency (lower bound) and a locked frequency (upper bound); if they do not alternate, the pass-band may be bounded by either of them [34]. The position of the pass-bands in the frequency domain can give information on the location of the system natural frequencies; in fact, they usually lie in, or at the boundary of, the pass-bands, even if cases have been noticed in which some frequency is in the attenuation zone [34].

The natural frequencies of the beam can be easily computed if the system transfer matrix $T^{N}$, relating state vectors to the ends of the $N$-bay chain, is evaluated. For this purpose, use can be made of the similarity transformation $T=\Psi \Lambda \Psi^{-1}$, where $\Psi$ is the matrix of the eigenvectors of $T$ and $\Lambda=\operatorname{diag}\left\{\sigma_{1} \sigma_{2}\right\}$. Then $T^{N}=\Psi \Lambda^{N} \Psi^{-1}$ can be found to be (see also reference [35] for an alternative derivation)

$$
T^{N}=\left[\begin{array}{cc}
\operatorname{Ch} N \mu+\frac{1}{2}\left(t_{11}-t_{22}\right) \frac{\operatorname{Sh} N \mu}{\operatorname{Sh} \mu} & \frac{\operatorname{Sh} N \mu}{\operatorname{Sh} \mu} t_{12} \\
\frac{\operatorname{Sh} N \mu}{\operatorname{Sh} \mu} t_{21} & \operatorname{Ch} N \mu+\frac{1}{2}\left(t_{11}-t_{22}\right) \frac{\operatorname{Sh} N \mu}{\operatorname{Sh} \mu}
\end{array}\right],
$$

where $\mathrm{Ch}=$ cosh and $\mathrm{Sh}=\sinh$. By equating separately to zero the coefficients of $T^{N}$, the characteristic equations in the unknown $\mu=\mu(\lambda)$ for four different boundary conditions (free-fixed, fixed-fixed, free-free and fixed-free) are obtained.

Here beams with both ends fixed, made of symmetrical elements, are considered, so that equations (A2)-(A4) yield

$$
\begin{gathered}
\operatorname{Re}(\mu)=0, \quad \operatorname{Im}(\mu)=j \pi / N, \quad j=1,2, \ldots, N-1, \\
t_{12} \equiv \alpha_{L R}-\alpha_{L L} \alpha_{R R} / \alpha_{L R}=0,
\end{gathered}
$$

in which, from equation (A3), $\operatorname{Im}(\mu)=\cos ^{-1}\left(\alpha_{L L} / \alpha_{R R}\right)$. Equations (A5) permit evaluation of natural frequencies $\lambda$. Note that $N-1$ frequencies lie in a pass-band (equation (A5a)) and one frequency lies on the boundary (equation (A5b)), it being equal to the frequency of the isolated locked element. The modal shape can be successively evaluated starting with the normalized state vector $\left\{\begin{array}{ll}0 & 1\end{array}\right\}^{\mathrm{T}}$ at the left of the chain and using the matrix $T^{k}$ to obtain displacement and force at the left of the $(k+1)$ th element: i.e., $u_{L, k+1}=$ $t_{12}$ Sh $k \mu / \mathrm{Sh} \mu$ and $f_{L . k+1}=\mathrm{Ch} k \mu, \mu$ being given by equations (A5).

\section{APPENDIX B: TRANSFER MATRIX OF THE THREE SUB-ELEMENTS UNIT}

By integrating equation (2) in the three individual sub-intervals of Figure 2 and using appropriate boundary conditions, the following receptances (see equation (A1) of 
Appendix A) are obtained:

$$
\begin{aligned}
& \beta_{L L}=\beta_{R R}=-\frac{1}{\beta \theta_{1}} \frac{1}{\tan \theta_{1} l_{1}}, \quad \beta_{L R}=-\frac{1}{\beta \theta_{1}} \frac{1}{\sin \theta_{1} l_{1}}, \\
& \gamma_{L L}=\gamma_{R R}=\left\{\begin{array}{ll}
\frac{1}{\beta \theta_{2}} \frac{1}{\tanh \theta_{2} l_{2}}, & \lambda<1 \\
-\frac{1}{\beta \theta_{2}} \frac{1}{\tan \theta_{2} l_{2}}, & \lambda>1
\end{array}\right\}, \\
& \gamma_{L R}=\left\{\begin{array}{ll}
\frac{1}{\beta \theta_{2}} \frac{1}{\sinh \theta_{2} l_{2}}, & \lambda<1 \\
-\frac{1}{\beta \theta_{2}} \frac{1}{\sin \theta_{2} l_{2}}, & \lambda>1
\end{array}\right\} .
\end{aligned}
$$

In equations (B1) $\beta_{i j}$ and $\gamma_{i j}$ refer to the sub-elements 1 and 2 , respectively, $l_{1}=2 \eta, l_{2}=$ $1 / 2-\eta$ are the sub-element lengths, and $\theta_{1}, \theta_{2}$ depend on $\lambda$ via equations (6).

By imposing equilibrium and compatibility at the joints between sub-elements, the receptances of the assemblage are obtained as

$$
\alpha_{L L}=\alpha_{R R}=\gamma_{L L}-\gamma_{L R}^{2} \frac{\beta_{L L}+\gamma_{L L}}{\left(\beta_{L L}+\gamma_{L L}\right)^{2}-\beta_{L R}^{2}}, \quad \alpha_{L R}=\frac{\beta_{L R} \gamma_{L R}^{2}}{\left(\beta_{L L}+\gamma_{L L}\right)^{2}-\beta_{L R}^{2}} .
$$

Finally, the transfer matrix $T$ of the unit can be built up as indicated in equation (A2) of Appendix A. Alternatively, $T$ can be obtained as the product of the transfer matrices $T_{j}(j=1,2,3)$ of the sub-elements, thus avoiding the use of equations (B2). 